\title{
Determinantes de la calidad de la información divulgada vía Web por las pequeñas y medianas cooperativas de Canarias
}

\author{
Zenona González Aponcio ${ }^{1}(0$ \\ Recibido: 1 de julio de 2019 / Aceptado: 20 de noviembre de 2019 / Publicado: 25 de febrero de 2020
}

\begin{abstract}
Resumen. El avance experimentado por las tecnologías de información, y en concreto el desarrollo de las tecnologías de Internet, ha tenido su máximo exponente en el despliegue progresivo de servicios y aplicaciones para las empresas, provocando que las pymes reaccionen ante la transformación digital de los negocios para adaptarse a las nuevas exigencias. Dentro de este grupo de empresas hay cooperativas que se enfrentan con mercados que requieren concepciones personalizadas y flexibles del producto o servicio. Ante estas exigencias, las cooperativas han de conectar con el entorno para captar las necesidades de clientes y socios, y obtener información que ayude a satisfacerlos. Para ello, resulta fundamental dotarse de sitios Web corporativos que generen información y conocimiento de apoyo al proceso de toma de decisiones y anticiparse a los cambios. El objetivo de este trabajo es conocer cuáles son los indicadores que explican la calidad de la información que divulgan las pequeñas y medianas cooperativas de Canarias a través del sitio Web corporativo. Para lograrlo, se basa en el modelo de Delone y McLean (2002 y 2003) adaptado a los sitios Web de 24 cooperativas que se identifican utilizando la base de datos SABI y cuyos datos se analizan aplicando el Modelo de Rasch (1980). Los resultados se corresponden con el total de cooperativas que tienen sitio Web activo y revelan que la calidad de la información que comparten las cooperativas mediante el sitio se basa más en información sobre los productos e información útil que en información para los socios y clientes, y algunos indicadores están condicionados por la provincia donde se localizan y el período en que se crean. Por consiguiente, conviene que las cooperativas no sólo valoren el mantener un sitio Web corporativo para compartir información completa, actualizada, relevante y exacta, sino también para comunicarse y realizar transacciones con los socios y los clientes que satisfagan sus necesidades y expectativas. Las conclusiones obtenidas demuestran que, en general, las pequeñas y medianas cooperativas seleccionadas usan sitios Web estáticos y estructurados sin que hayan evolucionado hacia portales empresariales, y que desaprovechan la información que pueden recoger del sitio para predecir e interpretar comportamientos de socios y clientes, así como para ofrecer productos y servicios ajustados a sus preferencias.
\end{abstract}

Palabras clave: Cooperativas; Tecnologías de Internet; Sitio Web; Información; Indicadores de la calidad. Claves Econlit: L25; O33; P13.

\section{[en] Determinants of the quality of information disclosed via the Web by small- and medium-sized cooperatives in the Canary Islands}

\begin{abstract}
The advance experienced by information technologies, and specifically the development of Internet technologies has had its maximum exponent in the progressive deployment of new services and applications for companies, which is leading SMEs to react to the digital transformation of business to adapt to new demands. Within this group of companies are cooperatives that face markets that require more personalized and flexible conceptions of the product or service. In response to these demands, cooperatives have to connect with the environment to capture the needs of customers and partners, and obtain information that helps to meet their needs. For this, it is essential that they are equipped with corporate Web sites that help to generate information and knowledge useful as a support to make decisions and anticipate changes. The objective of this work is to know which are the indicators that explain the quality of the information that small and medium-sized cooperatives in the Canary Islands disclose through the corporate website. To achieve this, it is based on the Delone and McLean model (2002 and 2003) adapted to the websites of 24 cooperatives that are identified using the SABI database and whose data are analyzed by applying the Rasch Model (1980). The results correspond to the total active cooperative Web site and reveal that the quality of information shared by cooperatives through the Web site is based more on information about products and useful information than on information for partners and customers, with some indicatos being conditioned by the province where they are located and the period in which they are created. Therefore, cooperatives should not only value maintaining a corporate website to share complete, up-to-date, relevant and accurate information, but also should communicate and carry out transactions with partners and customers that meet their needs and expectations. The conclusions obtained show that, in general, selected small and medium cooperatives use static and structured Web sites without having evolved towards business portals, and that they miss out on the information they can collect from the site to predict and interpret behaviors of partners and clients, as well as to offer products and services tailored to their preferences.
\end{abstract}

Keywords: Cooperatives; Internet technologies; Web site; Information; Quality indicators. 
Sumario. 1. Introducción. 2. Revisión teórica. 3. Modelo de investigación. 4. Metodología. 5. Análisis y resultados. 6. Discusión de los resultados. 7. Conclusiones e implicaciones. 8. Limitaciones y futuras líneas de investigación. 9. Referencias bibliográficas.

Cómo citar: González Aponcio, Z. (2020) Determinantes de la calidad de la información divulgada vía Web por las pequeñas y medianas cooperativas de Canarias. REVESCO. Revista de Estudios Cooperativos, vol. 133, e67340. https://dx.doi.org/10.5209/REVE.67340.

\section{Introducción}

La evolución natural de las políticas empresariales relacionadas con el uso de Internet comienza con la incorporación de tecnologías de la información básicas para desarrollar electrónicamente algunas actividades comerciales, después las empresas se esfuerzan en establecer iniciativas encaminadas a realizar transacciones comerciales en Internet y, finalmente, se plantean integrar sus actividades de comercio electrónico dentro de los procesos de negocio para encontrar alguna fuente de ventaja competitiva. En general, las pymes están presentes en Internet de dos formas, manteniendo un sitio Web corporativo estático sin apenas cambios en los contenidos informativos, y que resulta útil para crear una imagen y darse a conocer con el propósito de captar nuevos clientes, o bien transformando los procesos empresariales para tener un sitio Web donde colaborar con clientes y proveedores mediante la creación de redes y experimentar nuevas formas de hacer negocios.

Sin embargo, las pymes no siempre se benefician del potencial que tienen las tecnologías de la información, limitándose a emplear tecnologías informáticas tradicionales y servicios asociados a ellas, dejando a un lado la posibilidad de usarlas a nivel organizativo y comercial ya que se enfrentan a las barreras propias de su naturaleza, como la falta de experiencia y la baja capacidad en recursos humanos y financieros, y a los inconvenientes vinculados con el desarrollo de los mismos. Por ello, para ser competitivos y sobrevivir en el entorno actual, requieren de sistemas de información que contemplen las necesidades de información de los usuarios e incorporen tecnologías adecuadas para captar, analizar y extraer todo el potencial de los datos y obtener información útil que apoye la toma de decisiones. Ante esta situación, es necesario trabajar con métodos y herramientas de gestión que faciliten el cambio y que las pymes se adapten a los modelos de negocio predominantes. En este sentido, las pymes que persiguen eficiencias en sus procesos de negocio externos por medio de las tecnologías de Internet generan modelos que permiten el establecimiento de relaciones eficaces con proveedores, clientes, empleados, etc.

Las tecnologías Web han provocado avances significativos en los métodos que utilizan las pymes para comunicar, compartir conocimiento, gestionar información y digitalizar procesos. El trabajo de Gras, et al. (2019) considera que las decisiones favorecedoras de la digitalización de las pymes españolas dependen de distintos factores, entre los que están la disponibilidad de un sitio Web corporativo como medio para interactuar con el entorno virtual. Y concluyen que, a pesar del avance experimentado en los últimos años (un 83,2\% dispone de sitio), siguen necesitando desarrollar la transformación digital del negocio.

Al igual que el resto de pymes, las cooperativas recurren a las tecnologías de la información para disminuir los costes, añadir valor tanto a los productos y servicios como a los procesos, y para mejorar su posición en el mercado. La dinámica de estos cambios obliga a que las cooperativas conozcan sus necesidades de información, aprovechen las tecnologías de la información y aporten valor al negocio, así como satisfacer las demandas de los clientes y los socios. Desde esta perspectiva, es necesario que las cooperativas garanticen no sólo el acceso continuado, fluido y riguroso a la información mediante el uso de tecnologías de la información sino su control y dominio. A pesar de la dificultad para cambiar los modelos de negocio, con una adecuada gestión de la información que difunden en los sitios Web corporativos, las cooperativas pueden sacar partido a las tecnologías de Internet para aumentar su eficiencia en la mejora y creación de servicios, ampliar la función comercial y fortalecer las relaciones digitales con los socios y los clientes. Las cooperativas localizadas en regiones ultraperiféricas como Canarias tienen un hándicap añadido y es la dificultad para impulsar nuevas acciones y expandirse, por lo que la presencia en Internet es una alternativa óptima para llegar a personas y a mercados imposibles de acceder habitualmente.

Los sitios Web de las cooperativas ofrecen la posibilidad de convertirse en un medio de comunicación alternativo para alcanzar los objetivos, especialmente aquellos sobre la digitalización del negocio y, dada su importancia, es necesario hacer un seguimiento del sistema evaluando la calidad de la información que se publica y usar indicadores concretos como índices de la calidad del sitio porque afectan al éxito del mismo. El objetivo del presente trabajo es determinar los indicadores que explican la calidad de la información que divulgan las cooperativas de Canarias a través del sitio Web corporativo. Para lograrlo, se basa en el modelo de evaluación del éxito de los sistemas de información desarrollado por Delone y McLean (2002 y 2003) adaptado a los sitios Web de las 24 cooperativas que tienen sitio activo e identificadas en la base de datos SABI.

Por último, esta investigación se estructura en siete apartados además de la introducción y las referencias bibliográficas. El primero comprende la revisión de la literatura; el segundo, el modelo teórico propuesto; el 
tercero plantea la metodología seguida incluyendo el ámbito, la muestra, la medida de las variables y el modelo de Rasch; el cuarto presenta el análisis de los datos y de los resultados; el quinto muestra la interpretación y discusión de los resultados; el sexto propone las principales conclusiones obtenidas describiendo las posibles implicaciones; y, finalmente, el séptimo aclara las limitaciones del estudio y la propuesta de futuras líneas de investigación.

\section{Revisión teórica}

Desde de los años noventa se realizan múltiples estudios centrados en las características de los sitios Web corporativos de las empresas, señalando que el éxito de un sitio depende de la satisfacción de las expectativas de los usuarios y uno de los elementos que influye es el nivel de calidad de la información (Palmer, 2002, Argawal y Venkatesh, 2002). Las primeras investigaciones sobre los sitios Web corporativos consideraban que eran una única puerta de acceso a la información interna para los usuarios (Shilakes y Tylman, 1998, Reynolds y Koulopoulos, 1999) y el estudio de Benbya et al. (2004) defiende que son plataformas virtuales con sistemas y tecnologías de la información que integran información, procesos y servicios que ayudan a la consecución de los objetivos empresariales (Aneja et al., 2000; Detlor, 2000; Dias, 2001; Chan y Liu, 2007; y Daniel y Ward, 2005). Posteriormente, Halim, et al. (2016) analizan los factores de la calidad del sitio Web corporativo en un grupo de pymes concluyendo que los principales son el tiempo de respuesta, la disponibilidad de la información adecuada cuando se necesita y un servicio rápido.

Gargallo y Pérez (2009) señalan la necesidad de que las cooperativas reciban apoyo por las distintas administraciones públicas para elaborar y mantener los sitios Web corporativos. No obstante, anteriormente ya Mozas y Bernal (2004) investigan la utilización de Internet en el sector cooperativo español concluyendo que su uso ofrece ventajas a las cooperativas por ser organizaciones donde la comunicación es fundamental para conseguir los objetivos empresariales. A continuación, Estapé-Dubreuil y Torreguitart-Mirada (2014) encuentran que las pymes de economía social podrían alcanzar un nivel adecuado de presencia en Internet y mejorar la gestión interna gracias a la integración de la información y los procesos.

López-Becerra, et al. (2012) determinan en qué medida usan los sitios Web las cooperativas del sector agroalimentario, resultando que depende de la cualificación de la dirección, del tamaño de la empresa y de la actividad principal. Los autores afirman que las cooperativas estiman ventajoso su uso porque mejora las relaciones con los proveedores, con los clientes, con los socios propietarios y la calidad de los servicios. También López, et al. (2015) estudian el uso de diversas tecnologías de Internet en las empresas de economía social y destacan los beneficios debido a que son herramientas para la comunicación, el acceso a información y la gestión organizacional eficaz. Además, aseguran que ayudan a atender las demandas de socios, clientes y proveedores, pudiendo mantener la presencia online activa con un sitio Web que favorezca el desarrollo del negocio electrónico. Para Mozas, et al. (2015) la comercialización es uno de los principales desafíos a los que se enfrentan las entidades de economía social, y en especial las cooperativas, por lo que es necesario aprovechar por ejemplo el uso empresarial de Internet para añadir valor a los principios y valores que defienden.

El trabajo de Juliá, et al. (2004) es el primero que plantea conocer la divulgación de la información empresarial utilizando los sitios Web de las cooperativas españolas de diferentes sectores, y encuentran que la mayor parte dispone de un servicio de noticias sobre la empresa y el sector, y publica información referida a la actividad empresarial y a la calidad. Bernal y Mozas (2008) aplican el modelo de Delone y McLean (2002 y 2003) al análisis de los sitios Web corporativos de las cooperativas españolas desde la perspectiva de la calidad de la información y la calidad del sistema, exponiendo que actualizan poco la información, agregan especialmente información de la empresa y no aprovechan suficientemente el potencial de las tecnologías Web para fomentar la interacción con los clientes. Después, López et al. (2014) incluyen una muestra de cooperativas al evalúar la calidad del sitio Web corporativo de las empresas agroalimentarias y concluyen que existe un número elevado de sitios desactualizados y obsoletos, coincidiendo con Bernal y Mozas (2008) en que sólo un grupo minoritario de empresas han percibido las ventajas que les proporcionan las tecnologías Web.

Montegut, et al. (2013) estudian el uso de Internet y del comercio electrónico en las cooperativas agroalimentarias, encontrando que un gran porcentaje cuenta con sitio Web corporativo para darse a conocer gracias a la difusión de información acerca de la cooperativa y que actualizan cada cierto tiempo. Incluso comentan que las cooperativas desaprovechan sus posibilidades como canal alternativo para fines comerciales. Por otro lado, Muñoz y Tirado (2014) realizan un estudio de la responsabilidad social y la transparencia de las cooperativas agroalimentarias en el sitio Web corporativo, hallando que la mayoría de la información difundida está relacionada con la identificación del negocio, las marcas, los premios, los mercados, la localización y la forma jurídica.

Cristóbal, et al. (2016) analizan los sitios Web corporativos de las cooperativas agroalimentarias oleicas, confirmando que disponen de sitio Web corporativo a partir de ciertos niveles de facturación, socios o 
trabajadores, la información que difunden no está relacionada con su tamaño y es necesario fomentar la presencia online para diferenciarse y potenciar las ventas. Siguiendo la metodología eMICA (Extended Model of Internet Commerce Adoption) de Burgess, et al. (2011) para evaluar los sitios Web corporativos desde el nivel promocional básico hasta el nivel de los procesos transaccionales, Cristóbal, et al. (2017) investigan si los sitios de dichas cooperativas facilitan una comunicación eficaz con su público objetivo y se adaptan al comercio electrónico. Concluyen que las cooperativas son conscientes de la importancia de estar presentes en la Web, si bien convendría impulsar tanto la interacción y la colaboración online como la conectividad, y generar y compartir contenidos y conocimientos por parte de los usuarios. Para conseguirlo añaden que se deben incorporar en los sitios Web contenidos de información, sistemas de comunicación, aplicaciones para el comercio electrónico y para funciones adicionales.

Posteriormente, Bernal et al. (2018) usan el modelo eMICA para plantear un estudio que pruebe la relación entre la eficiencia del negocio y la calidad del sitio Web corporativo de las cooperativas de aceite de oliva, llegando a la conclusión de que no existe una relación directa entre estas variables y tener un sitio Web corporativo, independientemente de que sea de alta calidad, porque no siempre conduce a un mejor desempeño organizacional en términos de eficiencia económica. Igualmente demuestran que los factores determinantes de la calidad del sitio Web corporativo son una buena capacidad de procesamiento, una alta interactividad y la disponibilidad de enlaces a las redes sociales. Asimismo exponen que la cantidad de información disponible en el sitio Web corporativo tiene un papel secundario, mientras que la capacidad del sitio para comunicarse con los usuarios, completar sus transacciones y compartir información juegan un papel primordial.

Jorge, et al. (2019) estudian la presencia en Internet y los servicios Web ofrecidos por las cooperativas agroalimentarias españolas, asegurando que están rezagadas con respecto al resto de empresas en términos de presencia en Internet y las cooperativas grandes tienen mayor grado de transformación digital teniendo en cuenta el desarrollo del sitio Web corporativo, la presencia en Internet, el comercio electrónico y los servicios Web ofrecidos. La última investigación de Bernal et al. (2019) se apoya, entre otros, en el modelo de Delone y McLean (2002 y 2003) para identificar los factores que explican la calidad del sitio Web en las empresas de producción ecológica de aceite de oliva y su relación con los rendimientos empresariales, afirmando que tienen un importante déficit respecto de la calidad y que la calidad del sistema es mayor que la calidad de la información. No obstante, hallan reciprocidad entre los ingresos de explotación y la calidad del sitio, en especial con la calidad de la información.

En definitiva, existen pocos artículos científicos publicados hasta el momento acerca de la calidad de los sitios Web corporativos de las cooperativas españolas, y las investigaciones se han orientado a analizar y valorar las características del sitio para proponer mejoras que inciden en el uso adecuado de los mismos atendiendo a los objetivos fijados por las cooperativas según tamaño y actividad.

\section{Modelo de investigación}

La revisión bibliográfica ha evidenciado que la calidad de la información puede evaluarse usando distintos indicadores y se aplica un número limitado de modelos para analizar la información de los sitios Web de las cooperativas, por lo que este trabajo pretende contribuir con la adaptación del modelo conceptual propuesto por Delone y McLean (2002 y 2003) para descubrir los indicadores de la calidad de la información que se encuentran en los sitios Web corporativos de las corporativas de Canarias porque es comprensible, sencillo y cuenta con la aceptación general de los investigadores en el ámbito de los sistemas de información empresariales (Myers, 1999; Nguyen, et al., 2015; Gable et al. 2008; y Petter et al. 2008).

El modelo de Delone y McLean (2002 y 2003) ofrece una visión integrada del concepto de éxito en el uso de los sistemas de información y mide el impacto de los beneficios que dichos sistemas proporcionan a las empresas. Los autores definen seis variables: la calidad de la información (cuestiones sobre el contenido), la calidad del sistema (funcionalidad de la aplicación), la calidad del servicio (aspectos del servicio ofrecido), la intensión de uso y uso, la satisfacción del usuario y los beneficios netos. La Figura 1 muestra estas variables y sus interdependencias. El componente dimensión de la calidad lo conforman las tres primeras variables y, según Delone y McLean, (2016), han de medirse por separado porque influyen individualmente en el uso y en la satisfacción del usuario al ser determinantes de la percepción de utilidad y facilidad de uso. Por tanto, una disminución de la calidad de la información se traduce en una disminución de ambas, lo que implica una caída de los beneficios. Los autores sugieren la existencia de un ciclo que se inicia con la creación de un sistema de información con distintos grados de calidad de la información, calidad del sistema y calidad del servicio. A continuación, según la experiencia derivada de su uso, los usuarios expresan un determinado nivel de satisfacción, lo que provoca un impacto individual que se traduce en un efecto global sobre la empresa. 
Figura. 1. Modelo de éxito de los sistemas de información empresariales.

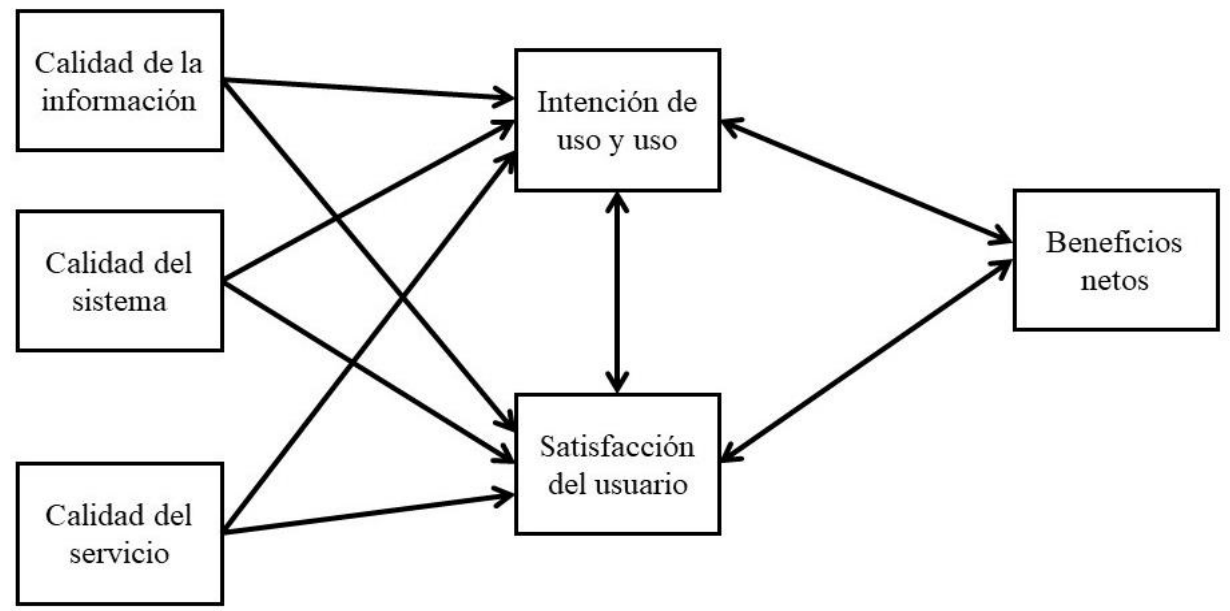

Fuente: Delone y McLean (2002 y 2003)

Petter, et al. (2012) afirman que la evaluación del éxito de los sistemas de información ha evolucionado, pasando de centrarse en mediciones cuantitativas y objetivas, a contemplar las consecuencias estratégicas y sociales del sistema con valoraciones cualitativas y subjetivas al reconocer la importancia que adquieren los clientes, los proveedores, los socios y las administraciones públicas, y que reflejan el efecto que tienen los sistemas de información desde una perspectiva individual y general.

En este estudio se analiza la calidad de la información, excluyendo el resto de componentes del modelo de éxito de los sistemas de información de Delone y McLean (1992 y 2003), quienes la definen como las características deseables de la salida de un sistema de información y un antecedente clave de la satisfacción del usuario. Estos autores proponen medirla en base a la calidad que produce el sistema y su utilidad para el usuario teniendo en cuenta que la información sea completa, oportuna, útil, relevante, con buena apariencia y formato, y fácil de comprender e interpretar. Anteriormente, Bailey y Pearson (1983) habían identificado la exactitud, la precisión, la actualidad, la disponibilidad, la confiabilidad, el formato, la relevancia y que sea completa y concisa. También ha quedado demostrado por Etezadi-Amoli y Farhoomand (1996), Seddon (1997) y Wixom y Watson (2001) que es una variable esencial, Ranganathan y Ganapathy (2002) consideran que es el determinante clave de la calidad de un sitio Web, y según Kim y Park (2013) representa la capacidad de comunicación más básica entre comprador y vendedor online $\mathrm{y}$, por tanto, se convierte en un factor fundamental para generar confianza.

Esta investigación pretende aportar conocimiento con respecto a los indicadores que delimitan la calidad de los contenidos informativos de los sitios Web de las cooperativas aplicando el modelo de Delone y McLean (2002 y 2003). La razón fundamental es que como la variable calidad de la información afecta al uso y a la satisfacción de los socios y clientes de las cooperativas, conviene medirla adecuadamente para conseguir el éxito del sitio Web corporativo ya que puede influir en la decisión final del socio y cliente y, por tanto, es necesario mejorar y mantener el nivel de calidad de la información que se publica con la definición de sus indicadores. El objetivo es conocer cuáles son los indicadores que explican la calidad de la información que divulgan las cooperativas a través del sitio Web, eligiendo para ello una muestra de pequeñas y medianas cooperativas situadas en la Comunidad Autónoma de Canarias.

\section{Metodología}

\section{1. Ámbito de la investigación}

El informe presentado por el Observatorio Nacional de las Telecomunicaciones y de la Sociedad de la Información u ONTSI (2018) referido a la implantación de las tecnologías de información en las pymes españolas recoge que el 77,7\% dispone de conexión a Internet y su propio sitio web corporativo. YanesEstévez, et al. (2019) analizan el comportamiento estratégico de las pymes en Canarias durante los años 2016, 2017 y 2018, llegando a concluir que evoluciona desde una postura de diferenciación en costes hacia la innovación. En este contexto, las tecnologías de Internet tienen un papel decisivo y los datos del INE (2018) permiten afirmar que las pymes utilizan el sitio Web corporativo fundamentalmente para darse a conocer y 
muy pocas personalizan el sitio teniendo en cuenta a los usuarios habituales $u$ ofrecen opciones para que los clientes puedan diseñar los productos.

La Comunidad Autónoma de Canarias es una región europea ultraperiférica, cuyo entorno insular influye considerablemente sobre las actividades que desarrolla el tejido empresarial, formado fundamentalmente por microempresas y, por lo tanto, ocupan una posición modesta en relación a media nacional respecto al uso de tecnologías de la información (INE, 2018). Según los últimos datos publicados por el Instituto Canario de Estadística o ISTAC (2010) presentan un alto grado de aplicación y penetración de tecnologías de información elementales, pero el equipamiento en otro tipo de tecnologías como aplicaciones ERP de gestión empresarial y soluciones CRM es mucho menor, siendo necesario realizar fuertes inversiones y convencer de los beneficios que aportan para obtener el máximo provecho de dichas tecnologías.

Asimismo, un 43,37\% usa Internet para disponer de sitio Web principalmente con el objetivo de presentar la empresa y publicar el catálogo de productos con los precios. Sin embargo, un porcentaje muy bajo usa el sitio Web corporativo para realizar pedidos y pagos, para hacer ofertas y recibir solicitudes de trabajo, para diseñar productos por clientes o personalizar el propio sitio para usuarios habituales al igual que el resto de pymes españolas. Esto significa que no sólo se enfrentan a impedimentos externos importantes, sino también a limitaciones internas que se generan básicamente por no contar con recursos suficientes para hacer frente a los cambios que supone realizar transacciones electrónicas, y planteamientos de una política de ampliación de mercados sin considerar la incorporación de tecnologías de la información. Evidentemente, estos resultados denotan deficiencias tanto en las condiciones tecnológicas como en la aplicación de tecnologías de Internet para apoyar la gestión de los clientes y de los proveedores.

De este modo, las tecnologías de Internet pueden representar para las pequeñas y medianas cooperativas de Canarias una opción óptima para acceder a recursos de información valiosos y a nuevos mercados sin las barreras de la insularidad. Según Román, et al. (2018), en Canarias hay un 35,1\% del total de cooperativas españolas constituidas que están situadas a gran distancia del resto de cooperativas españolas y se diferencian por especificidades productivo-insulares consecuencia de su posición geográfica y condición de archipiélago, por lo que la presencia en Internet mediante un sitio Web corporativo es vital. Los últimos datos publicados por el INE (2019) hacen referencia a que hay 465 sociedades cooperativas con la particularidad de que han desaparecido 167 en los últimos diez años, no existiendo estudios que se centren en algún aspecto sobre sus sitios Web corporativos.

\subsection{Muestra}

Para definir la población de las cooperativas de las dos provincias que constituyen la Comunidad Autónoma de Canarias (Las Palmas de Gran Canaria y Santa Cruz de Tenerife) se consulta la base de datos SABI (Sistema de Análisis de Balances Ibéricos) de la empresa E-informa. El total de cooperativas activas económicamente a fecha 12 de febrero de 2019 es de 170 .

Los criterios aplicados para seleccionar la muestra del estudio fueron que las cooperativas generen un volumen de negocio de entre 2 y 50 millones de euros y tengan entre 10 y 250 empleados, y que el sitio Web corporativo esté a pleno funcionamiento. Así, se consigue una muestra de 35 pequeñas y medianas cooperativas con sitio Web, de las cuales 24 poseen un sitio operativo y representan el $14.1 \%$ de la población total de cooperativas. La muestra final coincide con todas las pequeñas y medianas cooperativas que mantienen actualmente un sitio Web corporativo activo y desarrollan su actividad principal en sectores tan diversos como el agroalimentario, el transporte terrestre de pasajeros, el farmacéutico, etc.

El sistema de codificación para identificar a cada cooperativa consistió en asignar primero el código de la provincia (G, para la provincia de Las Palmas de Gran Canaria y T, para la provincia de Santa Cruz de Tenerife), a continuación el código correspondiente al tamaño ( $\mathrm{P}$, pequeñas y $\mathrm{M}$, medianas), luego el período de constitución (2, en los años 2000; 3, en los años 90; 4, en los años 80; 5, en los años 70; 6, en los años 60; 7 , en los años 50; y 8, a principios del siglo XX) y los dos últimos dígitos se corresponde con el número asignado a cada cooperativa.

\subsection{Medida de las variables}

Para concretar los determinantes de la calidad de la información del sitio Web corporativo de las pequeñas y medianas cooperativas de Canarias se utilizan diecisiete indicadores, agrupados en cuatro dimensiones que se obtienen de integrar en la variable sobre la calidad de la información que proponen Delone y McLean (2002) los indicadores que definen Cristóbal, et al. (2017) y Bernal et al. (2019).

La primera dimensión del estudio es la información de la cooperativa e incluye la misión, visión, valores, objetivos e historia, siendo muy habitual encontrarla en un sitio Web corporativo; la información de los proyectos actuales y futuros de la cooperativa, relevante para el usuario y reflejo de la preocupación por mantenerlos al día e informados; la información de la localización mediante un mapa como Google Maps, indicativo de que usan alguna tecnología colaborativa de la Web 2.0; la información de interés relativa a 
socios y clientes, de vital importancia para ofrecer un perfil estable y consolidado; e información de la política de privacidad para proteger y preservar los derechos de los usuarios. La segunda dimensión comprende la información del producto, en concreto las características, los servicios, las ofertas y promociones, y las normas de calidad. La tercera dimensión abarca la información del entorno que se publica para dar a conocer noticias y eventos del sector, temas relacionados con la actividad y, en especial, sobre su realidad y dimensión social, enlaces a sitios Web de interés para los usuarios y suscripción a revistas, boletines, etc. Y la cuarta dimensión está formada por un conjunto de características que definen la utilidad de la información, actualizada, completa, clara, comprensible y que aparece en un lugar destacado del sitio Web.

Los datos del sitio Web de cada cooperativa fueron recogidos durante la primera semana del mes marzo de 2019, observando detalladamente los contenidos informativos que divulgan y fijando el valor 1 si agregan los indicadores definidos y el valor 0 en caso contrario.

\subsection{Modelo de Rasch}

Para el análisis de los datos en relación a la calidad de la información de los sitios Web de las pequeñas y medianas cooperativas de Canarias se utiliza el Modelo de Rasch (1980) dicotómico porque garantiza unos resultados que reflejan la situación real del constructo analizado para muestras pequeñas (Linacre, 1999) y, al permitir representar en una única dimensión a cooperativas e indicadores, se puede hallar su dificultad y la probabilidad de que sean corroborados con éxito. Además, el constructo se representa en dicha dimensión situando simultáneamente cooperativas e indicadores para analizar las interacciones entre ambos; la puntuación de las cooperativas no depende de los indicadores y la distribución de la muestra no afecta a las estimaciones de sus parámetros; se consiguen resultados para diagnosticar comportamientos colectivos e individuales en el constructo cuando los datos están ajustados al modelo; y se puede verificar la presencia de equivalencias entre las cooperativas según tamaño, provincia y antigüedad.

Los datos se trataron mediante el programa informático Winsteps Rasch Measurement 3.92.1.0 (Linacre, 2014) porque es la solución adecuada para aplicar el Modelo de Rach (1980) dado que admite calcular al mismo tiempo la dificultad de los indicadores, el grado de ajuste de los datos al modelo, la probabilidad de respuesta de una cooperativa y los estadísticos generales (Oreja, 2015). Para comprobar si los datos se ajustan al modelo, se determina en primer lugar el INFIT ${ }^{2}$ o estadístico de ajuste interno, sensible a comportamientos inesperados (según la expectativa del modelo) que determina la presencia de indicadores cercanos al nivel de capacidad de las cooperativas, y en segundo lugar el OUTFIT $^{3}$ o estadístico de ajuste externo, sensible a comportamientos inesperados (según el modelo) que contribuye a la existencia de indicadores lejanos al nivel de capacidad de las cooperativas.

\section{Análisis y resultados}

\subsection{Análisis de los datos}

Una vez realizado el procesamiento de los datos, y considerando que se trata de una muestra pequeña, según los datos de la Tabla 1 se confirma el ajuste logrado ya que se presenta una fiabilidad de las medidas de las cooperativas que varía entre 0,75 para el valor real ${ }^{4}$ y 0,78 para el valor del modelo ${ }^{5}$. En el caso de los indicadores de la calidad de la información del sitio Web corporativo, la fiabilidad es de 0,81 para el valor real y 0,82 para el valor del modelo. Igualmente, la separación entre cooperativas y entre indicadores es baja, el coeficiente alfa de Cronbach (0.77) es aceptable según Linacre (2014), y los datos están completos porque el coeficiente de correlación de Pearson de las cooperativas e indicadores es muy bueno, lo que convierte en válidos los indicadores de la calidad de la información seleccionados para observar en los sitios Web de la muestra de cooperativas estudiada (Tabla 1). 
Tabla. 1. Fiabilidad, separación, correlación, INFIT y OUTFIT de cooperativas e indicadores en la variable latente.

\begin{tabular}{|c|c|c|}
\hline & Indicadores & Cooperativas \\
\hline Fiabilidad & & \\
Real & 0.81 & 0.75 \\
Modelo & 0.82 & 0.78 \\
\hline Separación & & \\
Real & 2.06 & 1.72 \\
Modelo & 2.16 & 1.86 \\
\hline Correlación & -1 & 0.99 \\
\hline INFIT & & \\
MNSQ & 0.96 & 0.98 \\
ZSTD & -0.1 & -0.1 \\
\hline OOUTFIT & & \\
MNSQ & 1.12 & 1.12 \\
ZSTD & 0.2 & 0 \\
\hline
\end{tabular}

Fuente: Tabla 3.1 del Winsteps y elaboración propia.

En definitiva, la clasificación de los indicadores según su dificultad y el nivel de la variable latente que explican, designada como indicadores de la calidad de la información de los sitios Web corporativos, son consistentes con el lugar que ocupa cada cooperativa analizada y proporciona una bondad de ajuste aceptable. El análisis de los estadísticos de validez INFIT y OUTFIT confirma el ajuste logrado, pues los indicadores y las cooperativas mantienen estadísticos admisibles a nivel de medias $\mathrm{MNSQ}^{6}$ porque están entre valores de 0.5 y 1.5 , lo que indica un ajuste entre los datos y el modelo sin que existan distorsiones, igual ocurre con las varianzas estándar $\mathrm{ZSTD}^{7}$ al tomar valores lógicos aceptables desde -2 hasta +2 . En definitiva, el grupo de pequeñas y medianas cooperativas de Canarias y los indicadores de la calidad de la información resultantes son fiables y válidos para el estudio.

\subsection{Resultados}

El mapa de Wright (1994) de cooperativas e indicadores resultante (Figura 2) refleja el rango de distribución de las 24 pequeñas y medianas cooperativas respecto de los indicadores de la calidad de la información del sitio Web corporativo, situándolas de menos a más de acuerdo con la medida respecto de la variable latente y ordenando los indicadores desde frecuentes hasta raros de encontrar en los sitios de la muestra. Así, la mayor presencia de la variable latente se localiza a la izquierda del mapa en el área comprendida entre la desviación estándar (S) respecto de la media de las cooperativas y, por consiguiente, representa a los sitios Web de las cooperativas donde se encuentra una cantidad considerable de indicadores de la calidad de la información (el $70,8 \%)$.

En la zona izquierda de la Figura 2, donde se posicionan la mayor parte de las cooperativas, su rango de distribución se solapa parcialmente con un área del rango de jerarquía de los indicadores en la parte derecha (en concreto nueve). La media (M) para las cooperativas es ligeramente menor que para los indicadores y el área de los indicadores difíciles de encontrar en los sitios Web corporativos está por encima del área de los parámetros del rasgo latente de las cooperativas, delimitado por la desviación típica (S). Esto indica que las cooperativas no incorporan dos indicadores de la calidad de la información (proyectos actuales y futuros, y suscripciones a revistas, boletines, etc.) y hay tres cooperativas de la provincia de Santa Cruz de Tenerife creadas en los años 70 (dos pequeñas y una mediana) que se sitúan por encima del resto de indicadores.

Al observar el rango de jerarquía de los indicadores de la calidad de la información, en el lado derecho de la Figura 2, se aprecia que la mitad de las cooperativas analizadas están considerablemente capacitadas en siete indicadores porque se encuentran situadas por encima de la media (M). Como los indicadores se muestran de menor a mayor dificultad, si el sitio Web corporativo contiene un indicador es porque también incluye los indicadores situados en los niveles inferiores de dificultad. Luego, el indicador bastante frecuente en los sitios Web de las cooperativas tiene que ver con la información del producto, y a continuación los indicadores que caracterizan a la utilidad de la información. 
Figura. 2. Mapa de Wright con la distribución de cooperativas e indicadores de la calidad de la información.

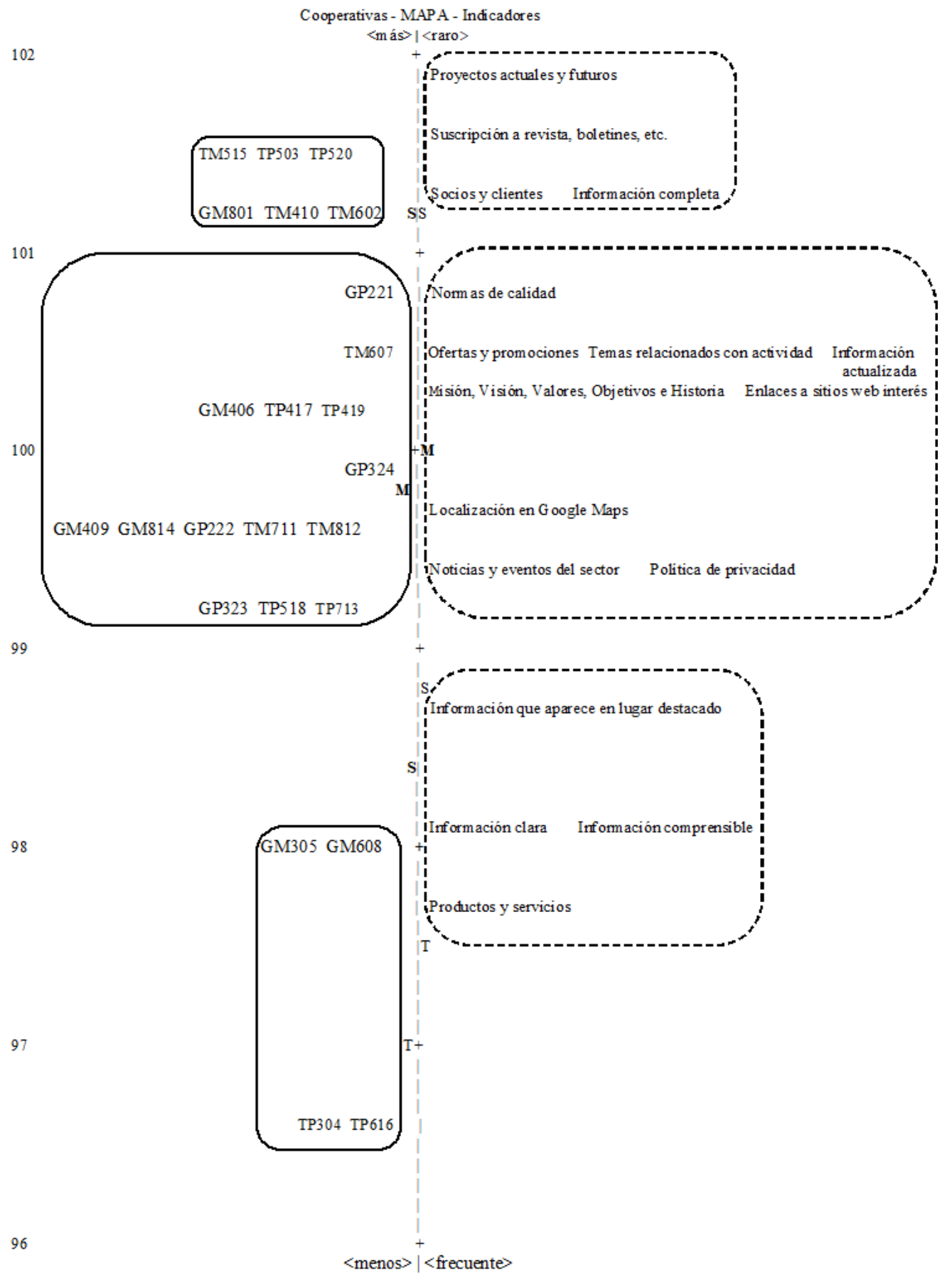

$\underline{\text { Estadístico }}$

M: Media

S: Desviación estándar

T: Doble de S

$\underline{\text { Provincia }}$

G: Las Palmas de Gran Canaria

T: Santa Cruz de Tenerife

Tamaño de las cooperativas

P: Pequeñas

M: Medianas
Período de constitución

2: años 2000

3: años 90

4: años 80

5: años 70

6: años 60

7: años 50

8: principios del siglo $\mathrm{XX}$

Fuente: Tabla 1 del Winsteps y elaboración propia.

Por consiguiente, en la Figura 2 resaltan tres grupos de cooperativas pequeñas y medianas en función del nivel de dificultad para albergar en el sitio Web corporativo indicadores de la calidad de la información. El primer grupo (TP304, TP616, GM305, GM608), situado por debajo de la S, está formado por las cooperativas que no incluyen indicadores en el sitio y por aquellas que poseen sitios menos preparados en 
indicadores de la calidad; el segundo grupo (GP323, TP518, TP713, GM409, GM814, GP222, TM711, TM812, GP324, GM406, TP417, TP419 y TM607, GP221) distribuido alrededor de la M y hasta las S, es numeroso y heterogéneo, e incluye a las cooperativas que son capaces de recoger en el sitio Web un número importante de indicadores, teniendo en cuenta que algunas aún necesitan añadir contenido informativo; y el tercer grupo (GM801, TM410, TM602, TM515, TP503 y TP520), localizado por encima de la S, lo constituyen las cooperativas que tienen sitios muy completos en indicadores de la calidad.

De igual forma, se pueden identificar tres grupos de indicadores de la calidad de la información que contienen los sitios Web de las pequeñas y medianas cooperativas de Canarias. En el primer grupo, situado entre la $\mathrm{S}$ y el doble de la desviación típica $(\mathrm{T})$, están los indicadores asociados con la información útil que se añade frecuentemente a los sitios Web y, por ende, en ellos se concentra la mayor experiencia de las cooperativas analizadas; el siguiente grupo, ubicado entre la $\mathrm{M}$ y las $\mathrm{S}$, está formado por los indicadores referidos a la dimensión del entorno y a la propia cooperativa; y finalmente, por encima de la $\mathrm{S}$ destaca un tercer grupo conformado por los indicadores que están muy poco presentes en los sitios Web y no pertenecen a la dimensión del producto.

Asimismo, al revisar individualmente la posición de las cooperativas respecto de los indicadores de la calidad de la información o viceversa, en el mapa de Wright de la Figura 2 se observan las siguientes situaciones que se repetirían para las cooperativas localizadas en la misma posición:

- Las cooperativas posicionadas en la zona baja del mapa no superan los indicadores definidos y tienen menor medida de la variable latente. Por el contrario, las ubicadas en la parte alta alcanzan todos los indicadores y la mayor medida.

Los sitios Web de las cooperativas GM305 y GM608 sólo tienen el indicador de productos y servicios, debido a lo cual están en posición de desventaja respecto del resto de cooperativas.

Un porcentaje importante de indicadores están agrupados cerca de la M de las cooperativas, luego éstas valoran sobre todo esa parte de la variable latente.

$\mathrm{Al}$ quedar libre la $\mathrm{M}$ de los indicadores, puede que sea necesario definir nuevos indicadores para estimar la parte superior e inferior de dicha variable. Si se presenta un vacío desde la perspectiva de las cooperativas, como en el caso de la GP324, quiere decir que hay muy pocos indicadores habituales en el sitio Web corporativo. No obstante, la medida obtenida es correcta y eficaz puesto que apenas se dan redundancias en los indicadores de la calidad y hay diferenciación suficiente entre las medidas de las cooperativas para poder distinguirlas.

Para conocer las diferencias entre las cooperativas en los indicadores de la calidad de la información del sitio Web por provincia, tamaño y período de constitución, se obtiene el Funcionamiento Diferencial del Ítem (DIF). Es decir, un indicador funcionaría diferencialmente cuando dos grupos de cooperativas lo contemplan de manera distinta, lo que implica que hay diferencia en la probabilidad de que consideren un indicador en particular y uno de los dos grupos tiene ventaja relativa respecto al otro. Al grupo aventajado se le denomina mayoritario o de referencia, mientras que el grupo perjudicado se conoce como minoritario o focal, comparable con el grupo de referencia.

Con el fin de evaluar el DIF, y asumir que las varianzas de cada subgrupo de cooperativas son distintas, se tienen en cuenta los resultados del contraste DIF en los niveles de dificultad de los indicadores entre las dos provincias, entre pequeñas y medianas, y entre los siete períodos de creación; y los valores de la $t$ y la $p$ del Test de Welch para aceptar o rechazar la hipótesis de igualdad de varianzas. De esta manera, cuando el contraste del DIF es menor a $0.43 \operatorname{logits}(\mathrm{p}<0.05$, y valor $\mathrm{t} \geq 2$ ), se trata de un funcionamiento diferencial insignificante; cuando es mayor o igual a 0.43 logits pero menor o igual a 0.64 logits, es moderado; y cuando es $>0.64 \operatorname{logits}(\mathrm{p}<0.05$, y valor $\mathrm{t} \geq 2)$ es severo.

En consecuencia, se determina el impacto de los indicadores que presentan DIF dentro de los grupos de cooperativas analizadas, y los resultados muestran que no existe desigualdad entre las cooperativas de Canarias de acuerdo con el tamaño respecto de los indicadores de la calidad de la información que aparecen en el sitio Web corporativo, pero sí se dan divergencias teniendo en cuenta la provincia donde se localizan y para dos períodos de constitución, los años 60 y los años 80. Según la Tabla 2, la diferencia es severa en la información que publican sobre la política de privacidad por provincias y extremadamente severa en la referida a la misión, visión, valores, objetivos e historia por periodos de constitución. 
Tabla. 2. DIF entre cooperativas por provincia y período de constitución.

\begin{tabular}{|c|c|c|}
\hline & Provincia & \multicolumn{1}{|c|}{ Período de constitución } \\
\hline Contraste DIF & -2.5 & -4.6 \\
\hline $\boldsymbol{t}$ & -2.38 & \multicolumn{1}{|c|}{0.49} \\
\hline $\boldsymbol{p}$ & 0.03 & 0.047 \\
\hline Indicador & Política de privacidad & $\begin{array}{l}\text { Misión, visión, valores, objetivos e } \\
\text { historia }\end{array}$ \\
\hline
\end{tabular}

Fuente: Tabla 30 del Winsteps y elaboración propia.

Por último, una vez hallado el DIF y para valorar el nivel de experiencia de las cooperativas integrando indicadores de la calidad de la información en el sitio Web por provincia y según los dos períodos de constitución anteriores, se examinan los resultados de la separación y la fiabilidad de los datos. De esta forma, en la Tabla 3 se aprecia que las cooperativas situadas en la provincia de Santa Cruz de Tenerife y las cooperativas de Canarias fundadas en los años 60 cuentan con sitios Web corporativos más ajustados a los indicadores de la calidad de la información que el resto de cooperativas de la muestra.

Tabla. 3. Experiencia de las cooperativas integrando indicadores por provincia y período de constitución.

\begin{tabular}{|c|c|c|c|c|}
\hline & \multicolumn{2}{|c|}{ Provincia } & \multicolumn{2}{c|}{ Período de constitución } \\
\hline & $\begin{array}{c}\text { Las Palmas de } \\
\text { Gran Canaria }\end{array}$ & $\begin{array}{c}\text { Santa Cruz de } \\
\text { Tenerife }\end{array}$ & Años 60 & Años 80 \\
\hline Separación & 1.14 & 1.96 & 2.09 & 0 \\
Real & 1.28 & 2.11 & 2.23 & 0 \\
\hline Modelo & & & & \\
Fiabilidad & 0.56 & 0.79 & 0.81 & 0 \\
Real & 0.62 & 0.82 & 0.83 & 0 \\
Modelo & & & \\
\hline
\end{tabular}

Fuente: Tabla 28 del Winsteps y elaboración propia.

\section{Discusión de los resultados}

De los resultados obtenidos se desprende que, en general, es importante decidir qué contenidos informativos son los apropiados para incorporar y difundir a través del sitio Web corporativo de las pequeñas y medianas cooperativas de Canarias porque de ello dependerán las visitas y las transacciones online.

El grupo de cooperativas que añade al sitio Web numerosos indicadores de la calidad de la información está caracterizado porque se constituyen en los años 70, están distribuidas uniformemente por tamaño y por provincias y, a su vez, dentro de cada provincia (alrededor de un 30\%). El resto de cooperativas divulga información relacionada con los indicadores en menor medida, y sólo dos cooperativas pequeñas creadas durante los años 60 y 90 en la provincia de Santa Cruz de Tenerife no completan el sitio Web con este tipo de contenidos. Sin embargo, en esta misma provincia aparece un mayor número de indicadores de la calidad de la información que en los sitios de las cooperativas de la provincia de Las Palmas de Gran Canaria. Con los sitios Web de las cooperativas fundadas en los años 60 ocurre lo mismo respecto de las constituidas en los años 80, sin que existan desajustes en el resto de períodos contemplados. Al mismo tiempo, la información de la política de privacidad es el indicador que distingue a las pequeñas y medianas cooperativas por provincia y, considerando los dos períodos anteriores de creación de las cooperativas, la desigualdad se encuentra en el indicador de la información completa.

Efectivamente, las cooperativas estudiadas publican en sus sitios Web mayormente información de los productos y los servicios, a continuación, se centran en contenidos con información clara, comprensible y que aparece en un lugar destacado del sitio, si bien es escasa la información destinada a socios y clientes. Concretamente, el $25 \%$ de las pequeñas y medianas cooperativas divulga información de los socios y clientes, y mantiene completa la información que aloja en el sitio. Por el contrario, consideran inoportuno 
tanto publicar información de sus proyectos actuales y futuros en este medio de comunicación alternativo como que los usuarios se suscriban a alguna revista o boletín propio.

En definitiva, como afirman Montegut et al. (2013), los indicadores de la calidad de la información del sitio Web de las cooperativas se basan menos en información para mejorar el nivel de satisfacción de socios y clientes. Incluso, algunos indicadores están condicionados por la situación geográfica y el año de fundación de las cooperativas. Esta situación puede deberse, por un lado, a la falta de recursos para invertir en la adquisición y gestión de tecnologías más avanzadas como las aplicaciones Web 2.0 y 3.0 y, por otro, a la necesidad de adquirir conocimientos para utilizar y explotar las prestaciones de dichas tecnologías. Consecuentemente, las posibilidades de las cooperativas analizadas son limitadas para integrar y asumir internamente los cambios que supone el comercio electrónico y su derivación en negocio electrónico.

Los resultados del estudio coinciden con Bernal, et al. (2018) y, por consiguiente, conviene que las cooperativas cuenten con un sitio Web corporativo para compartir información completa, actualizada, relevante, exacta, detallada y fácil de interpretar, principalmente para obtener información en el proceso de retroalimentación del sistema que les permita tomar decisiones adecuadas. De acuerdo con Bernal y Mozas (2008) y López, e al. (2014), además de mantener actualizada y completa la información, es oportuno que las cooperativas mejoren el contenido informativo del sitio Web incluyendo, por ejemplo, información sobre la misión, visión, valores y objetivos en la presentación de la cooperativa; acceso a portales especializados; información de ofertas y promociones puntuales; información de temas relacionados con la actividad empresarial y el entorno; información de normas de calidad que afecten a los productos y a los servicios; e información dirigida en exclusiva a clientes y socios.

En resumen, los sitios Web corporativos de las pequeñas y medianas cooperativas de Canarias son una alternativa que contribuye a la desaparición de las barreras tradicionales como la lejanía de proveedores y clientes, y a difundir nuevas oportunidades de negocio. Entre las ventajas que podrían conseguir las cooperativas usando este cauce se encuentran la mejora de las comunicaciones con los clientes y socios; la promoción de los productos, los servicios y la imagen corporativa; disponer de una alternativa de comercialización sin la limitación de la insularidad; y realizar la distribución de los productos por medio de una nueva vía libre de barreras geográficas.

\section{Conclusiones e implicaciones}

Las principales conclusiones que se pueden establecer sobre los indicadores de la calidad de la información incluidos en los sitios Web corporativos de las pequeñas y medianas cooperativas de Canarias son las siguientes:

- Existen pocas cooperativas con sitio Web activo, y las cooperativas que lo tienen se preocupan en especial de la utilidad de la información que divulgan por este canal.

Es necesario mentalizar a los responsables de las cooperativas de la conveniencia de utilizar el sitio Web no sólo para mejorar la comunicación y la imagen corporativa, sino incluso para recoger y analizar información que genere nuevos conocimientos acerca de los socios y de los clientes, y de este modo propiciar su familiarización con productos y servicios, y predecir e interpretar comportamientos con el propósito de ajustarlos a las preferencias de cada grupo.

- Las cooperativas mejor posicionadas en indicadores de la calidad de la información se crearon hace prácticamente sesenta años y, por tanto, no puede afirmarse que las cooperativas fundadas durante la era digital estén mejor preparadas en la dimensión de calidad de la información para conseguir el éxito del sitio Web corporativo.

- La situación geográfica afecta a los indicadores de la calidad de la información de los sitios Web, al contrario de lo que ocurre con el tamaño de las cooperativas.

- Las cooperativas se encuentran rezagadas en el aprovechamiento de la información que ofrecen y recogen en el sitio Web, por lo que sería beneficioso que los organismos y asociaciones competentes propicien un escenario asumiendo el papel determinante para romper la brecha y que las cooperativas puedan beneficiarse de las ventajas.

En absoluto se pueden definir estrictamente los sitios Web de las cooperativas como portales empresariales donde desarrollan parte del negocio, sino que predominan los sitios estáticos para darse a conocer en Internet y los sitios algo estructurados que facilitan y agilizan la búsqueda de información a los ususarios, quienes tienen la opción de ponerse en contacto con las cooperativas esencialmente por correo electrónico, y éstas suelen actualizar la información sin renovar muchos contenidos. Por tanto, las posibilidades de las cooperativas son muy limitadas para incorporar y asumir internamente los cambios que conlleva el negocio electrónico. 
Respecto de las implicaciones empresariales, esta investigación ayuda a que los sitios Web corporativos se perciban como una oportunidad para mejorar la digitalización de las pequeñas y medianas cooperativas de Canarias, y, contribuye a concienciar a los organismos implicados de la importancia de adoptar posturas proactivas que incluyan políticas transversales tales como la formación, dada la escasa preparación en gestionar recursos de sistemas y tecnologías de la información, la mejora de la infraestructura tecnológica necesaria, la incorporación de dinamizadores de la innovación, apoyos para crear nuevos desarrollos comerciales, etc. Estas acciones podrían dar lugar a una mejora equilibrada de los contenidos informativos de los sitios Web de las cooperativas ya que la diferenciación en términos de la calidad de la información ni mucho menos es una característica en exclusiva del resto de empresas.

Para finalizar, es el único estudio que, partiendo de los datos recogidos en los sitios Web corporativos de las pequeñas y medianas cooperativas de Canarias, permite tener una visión general de los indicadores de la calidad de la información que difunden por este sistema, y las conclusiones obtenidas pueden ayudar a los responsables de las cooperativas a tomar decisiones para mejorar la calidad del sitio y su éxito.

\section{Limitaciones y futuras líneas de investigación}

Los resultados del presente trabajo se encuentran condicionados por los tipos de indicadores de la calidad de la información definidos, y por el tamaño y la heterogeneidad de las actividades de las cooperativas seleccionadas. Por tanto, las futuras líneas de investigación deben orientarse a superar las limitaciones indicadas incluyendo los sectores de actividad, otros indicadores para profundizar en el análisis y el resto de componentes de la dimensión de la calidad propuesta por Delone y McLean (2002 y 2003), la calidad del sistema y la calidad del servicio del sitio Web corporativo.

\section{Referencias bibliográficas}

Aneja, A., Rowan, C. y Brooksby, B. (2000) Corporate Portal Framework for Transforming Content Chaos on Intranets. Intel Technology Journal, Vol. 4, $\mathrm{N}^{\mathrm{o}}$ 1, pp. 1-7.

Argawal, R. y Venkatesh, V. (2002) Assesing a firm's Web presence: a heuristic evaluation procedure for the measurement of usability. Information Systems Research, Vol. 13, No 2, pp. 168-186.

Bailey, J. E. y Pearson, S. W. (1983) Development of a Tool for measuring and Analyzing Computer User Satisfaction. Management Science, Vol. 29, No 5, pp. 530-545. DOI: 10.1287/mnsc.29.5.530.

Benbya, H., Passiante, G. y Belbaly, N. A. (2004) Corporate portal: A tool for knowledge management synchronization. International Journal of Information Management, Vol. 24, $\mathrm{N}^{\circ} 3, \quad$ pp. 201-220. DOI: 10.1016/j.ijinfomgt.2003.12.012.

Bernal, E. B. y Mozas, A. (2008) Evaluación del uso comercial de la World Wide Web por parte de las cooperativas de segundo grado españolas. Revista Española de Estudios Agrosociales y Pesqueros, Vol. 219, pp. 181-200.

Bernal, E. B., Mozas, A, Fernández, D. y Puentes, R. (2019) Calidad de los sitios Web corporativos en el sector agroalimentario ecológico y sus factores explicativos: el papel del cooperativismo. CIRIEC-España, Revista de Economía Pública, Social y Cooperativa, № 95, pp. 95-118. DOI: 10.7203/CIRIEC-E.95.13207.

Bernal, E. B., Mozas, A., Medina, M. J. y Fernández, D. (2018) Evaluation of Corporate Websites and Their Influence on the Performance of Olive Oil Companies. Sustainability, Vol. 10, pp. 1274-1285. DOI:10.3390/su10041274.

Burgess, L., Parish, B. y Alcock, C. (2011) To what extent are regional tourism organisations (RTOs) in Australia leveraging the benefits of web technology for destination marketing and eCommerce?. Electronic Commerce Research, Vol. 11, № 3, pp. 341-355.

Chan, E. H. y Liu, C. (2007) Corporate portals as extranet support for the construction industry in Hong Kong and nearby regions of China. Journal of Information Technology in Construction (ITcon), Vol. 12, $\mathrm{N}^{\circ} 12$, pp. 181-192.

Cristóbal E., Montegut Y. y Gómez M. J. (2016) Factores determinantes del uso de Internet en la comercialización del aceite de oliva. Análisis del sector en Cataluña. REVESCO. Revista de Estudios Cooperativos, Segundo Cuatrimestre, $\mathrm{N}^{\circ}$ 121, pp. 33-61. DOI: https://doi.org/10.5209/rev_REVE.2016.v121.51308.

Cristóbal, E., Montegut, Y. y Daries, N. (2017) Cooperativismo 2.0: presencia en Internet y desarrollo del comercio electrónico en las cooperativas oleícolas de Cataluña. REVESCO, Revista de Estudios Cooperativos, Segundo Cuatrimestre, No 124, pp. 47-73. DOI: https://doi.org/10.5209/REVE.54926.

Daniel, E. y Ward, J. (2005) Enterprise Portals: Addressing the Organizational and Individual Perspectives of Information Systems. ECIS 2005 Proceedings. Vol. 3, pp. 26-28.

Delone, W. H., y McLean, E. R. (1992) Information systems success: The quest for the dependent variable. Information Systems Research, Vol. 3, № 1, pp. 60-95. DOI: 10.1287/isre.3.1.60.

Delone, W. H., y McLean, E. R. (2002) Information Systems Success Revisited. Proceedings de 35th Annual Hawaii International Conference on System Sciences, pp. 2966-2976. IEEE. DOI: 10.1109/HICSS.2002.994345.

Delone, W. H., y McLean, E. R. (2003) The Delone and McLean Model of Information Systems Success: A Ten-Year Update, Journal of Management Information Systems, Vol. 19, $\mathrm{N}^{\mathrm{o}}$ 4, pp. 9-30. DOI: 10.1080/07421222.2003.11045748. 
Delone, W. H. y McLean, E. R. (2016) Information systems success measurement. Foundations and Trends. Information Systems, Vol. 2, No 1, pp. 1-116. DOI: 10.1561/2900000005.

Detlor, B. (2000) The Corporate Portal as an Information Infrastructure: Towards a Framework for Portal Design. International Journal of Information Management, Vol, 20, $\mathrm{N}^{\circ}$ 2, pp. 91-101. DOI: 10.1016/S0268-4012(99)000584.

Dias, C. (2001) Corporate portals: a literature review of a new concept in Information Management. International Journal of Information Management, Vol. 21, No 4, pp. 269-287. DOI: 10.1016/S0268-4012(01)00021-4.

Estapé-Dubreuil, G. y Torreguitart-Mirada C. (2014) Firm-Level Evidence of ICT Adoption among SMEs of the Social Economy in Spain. Journal of Electronic Commerce in Organizations, Vol 12, $\mathrm{N}^{\mathrm{o}} 1$, pp. 13-64. DOI: 10.4018/jeco.2014010102

Etezadi-Amoli, J. y Farhoomand, A. F. (1996) A structural model of end user computing satisfaction and user performance. Information and Management, Vol. 30, No 2, pp. 65-73. DOI: 10.1016/0378-7206(95)00052-6.

Gable, G. G., Sedera, D. y Chan, T. (2008) Re-conceptualizing information system success: The IS-impact measurement model. Journal of the Association for Information Systems, Vol. 9, № 7, pp. 377-408.

Gargallo, A. y Pérez, J. (2009) El papel de las tecnologías de la información y la comunicación en las empresas de economía social. REVESCO. Revista de Estudios Cooperativos, Primer Cuatrimestre, № 97, pp. 90-116.

Gras, J. M. G., Solves, I. M., De la Cruz, M. E. y Mateo, J. M. (2019) Informe de la PYME 2018. Comunidad Valenciana.: Análisis estratégico para el desarrollo de la PYME: digitalización y responsabilidad. Universidad Miguel Hernández. 90 p.

Halim, E., Setiawan, D. P. y Novela, E. S. (2016) Factors affect quality of SMEs' online marketing website based on DeLone and McLean Model. En International Conference on Information Management and Technology (ICIMTech) (pp. 338-343). IEEE. DOI: 10.1109/ICIMTech.2016.7930357.

INE (2018) Encuesta de uso de TIC y Comercio Electrónico (CE) en las empresas 2017-2018. Documento disponible en https://www.ine.es (consulta: 22 de marzo de 2019).

INE (2019) Estadística de sociedades mercantiles. Documento disponible en https://www.ine.es (consulta: 22 de marzo de 2019).

ISTAC (2010) Encuesta de tecnologías de la información y la comunicación en las empresas de canarias 2009/2010. Estadísticas económicas. Documento disponible en http://www.gobiernodecanarias.org/istac (consulta: 11 de abril de 2019).

Jorge, J., Chivite, M. P. y Salinas, F. (2019) La transformación digital en el sector cooperativo agroalimentario español: situación y perspectivas. CIRIEC-España, Revista de Economía Pública, Social y Cooperativa, No 95, pp. 39-70. DOI: 10.7203/CIRIEC-E.95.13002.

Juliá, J. F., García, G. y Polo, F. (2004) La información divulgada a través de internet por las cooperativas. CIRIECEspaña, Revista de Economía Pública, Social y Cooperativa, № 49, pp. 167-192.

Kim, S. y Park, H. (2013) Effects of various characteristics of social commerce (s-commerce) on consumers' trust and trust performance. International Journal of Information Management, Vol. 33, $\mathrm{N}^{\mathrm{o}}$ 2, pp. 318-332. DOI: 10.1016/j.ijinfomgt.2012.11.006.

Linacre, J. M. (1999) Understanding Rasch measurement: estimation methods for Rasch measures. Journal of outcome measurement, Vol. 3, pp. 382-405.

Linacre, J. M. (2014) Winsteps (Version 3.81. 0). Beaverton, Oregon: Winsteps.com.

López, E. I., Arcas, N. y Alcón, F. (2014) Uso y calidad de los sitios Web corporativos: evaluación en las empresas agroalimentarias murcianas. Revista Española de Estudios Agrosociales y Pesqueros, № 237, pp. 155-179.

López, E. I., Arcas, N., Alcón, F. y Guerola, R. (2015) De la teoría a la práctica. Uso de las TIC relacionadas con internet por la economía. En Arcas, N., Alcón, F. y López, E.I. (coordinadores) La economía social y los negocios online. Tendencias y claves del éxito, Serie Economía, Vol. 25, pp. 87-130, Edita: Cajamar Caja Rural.

López-Becerra, E. I., Arcas-Lario, N. y Alcón-Provenci, F. (2012) Los sitios Web corporativos de las cooperativas agroalimentarias. Antecedentes y consecuencias de su adopción. CIRIEC-España, Revista de Economía Pública, Social y Cooperativa, $\mathrm{N}^{\mathrm{o}} 76$, pp. 261-282.

Montegut, Y., Cristóbal, E. y Gómez, M.J. (2013) La implementación de las TIC en la gestión de las cooperativas agroalimentarias: el caso de la provincia de Lleida. REVESCO. Revista de Estudios Cooperativos, Primer Cuatrimestre, $\mathrm{N}^{\circ} 110$, pp. 223-253. DOI: 10.5209/rev_REVE.2013.v110.41442.

Mozas, A. y Bernal, J. E. (2004) Integración cooperativa y TIC's: presente y futuro. CIRIEC España. Revista de Economía Pública, Social y Cooperativa, № 49, pp. 143-166.

Mozas, A., Bernal, E., Medina, M. J. y Fernández, D. (2015) Internet: un mundo de posibilidades para las entidades de la economía social. En Arcas, N., Alcón, F. y López, E.I. (coordinadores) La economía social y los negocios online. Tendencias y claves del éxito, Serie Economía, Vol. 25, pp. 53-70, Edita: Cajamar Caja Rural.

Muñoz, R. y Tirado, P. (2014) Responsabilidad social y transparencia a través de la Web: un análisis aplicado a las cooperativas agroalimentarias españolas. REVESCO. Revista de Estudios Cooperativos, Primer Cuatrimestre, $\mathrm{N}^{\mathrm{o}}$ 114, pp. 84-105. DOI: 10.5209/rev_REVE.2014.v114.44293.

Myers, M. D. (1999) Investigating information systems with ethnographic research. Communications of the Association for Information Systems, Vol. 2, No 1, pp. 23.

Nguyen, T. D., Nguyen, T. M., y Cao, T. H. (2015) Information Systems Success: A Literature. FDSE 2015 Proceedings, Vol. 9446, pp. 242. DOI:10.1007/978-3-319-26135-5_18.

ONTSI (2018) ePyme 17. Análisis sectorial de implantación de las TIC en las empresas españolas. Observatorio Nacional de las telecomunicaciones y de la sociedad de la información. Ministerio de Economía y Empresa. Gobierno de España. Documento disponible en https://www.ontsi.red.es (consulta: 11 de abril de 2019). 
Oreja-Rodríguez, J. R. (2015) Mediciones, Posicionamientos y Diagnósticos Competitivos. Santa Cruz de Tenerife: Instituto Universitario de la Empresa (IUDE) de la Universidad de La Laguna.

Palmer, J. (2002) Web site usability, design and performance metrics. Information System Research, Vol. 13, No 2, pp. 151-167.

Petter, S., Delone, W. y McLean, E. (2008) Measuring information systems success: Models, dimensions, measures, and interrelationships. European Journal of Information Systems, Vol. 17, $\mathrm{N}^{\mathrm{o}} \quad 3, \quad \mathrm{pp} .236-263$. DOI:10.1057/ejis.2008.15.

Petter, S., Delone, W. y McLean, E. R. (2012) The past, present, and future of IS Success. Journal of the Association for Information Systems, Vol. 13, No 5, pp. 341-362.

Ranganathan, C. y Ganapathy, S. (2002) Key dimensions of business-to-consumer web sites. Information and Management, Vol. 39, No 6, pp. 457-465. DOI: 10.1016/S0378-7206(01)00112-4.

Rasch, G. (1980) Probabilistic models for some intelligence and attainment tests. Copenhagen, Denmark: Danish Institute for Educational Research.

Reynolds, H. y Koulopoulos, T. (1999) Enterprise knowledge has a face. Intelligent Enterprise, Vol. 2, № 5, pp. 29-34.

Román, C., Galván, I. y Domínguez, M. P. (2018) Los principales aspectos jurídico-económicos del proyecto de ley de sociedades cooperativas de Canarias. CIRIEC-España. Revista Jurídica de Economía Social y Cooperativa, № 32 , pp. 9-36.

Seddon, P. B. (1997) A respecification and extension of the Delone and McLean model of IS success. Information systems research, Vol. 8, No 3, pp. 240-253. DOI: 10.1287/isre.8.3.240.

Shilakes, C. C. y Tylman, J. (1998) Enterprise information portals. New York: Merril Lynch.

Wixom, B. H. y Watson, H. J. (2001) An Empirical Investigation of the Factors Affecting Data Warehouse Success. MIS Quarterly, Vol. 25, No 1, pp. 17-41. DOI: 10.2307/3250957.

Wright, B. (1994) Reasonable mean-square fit values. Rasch Meas Trans, Vol. 8, № 3, p. 370.

Yanes-Estévez, V, García-Pérez, A. M. y Oreja-Rodríguez, J. R. (2019) La dinámica del comportamiento estratégico de las pymes. El caso de Canarias. Emprendimiento y Negocios Internacionales, Vol. 3, No 2, pp. 26-37. 